\title{
«LENTICCHIE E SALUMI»: L'EKPHRASIS NEGLI STORICI GRECI
}

\author{
«LENTILS AND SAUSAGES»: \\ EKPHRASIS IN THE GREEK HISTORIANS
}

\author{
Francesco De Martino \\ Università degli Studi di Foggia \\ frademartino@alice.it
}

DOI: $10.1387 /$ veleia.14971

\begin{abstract}
Riassunto: Come mostrano numerose testimonianze, la storiografia è il genere più congeniale — persino più dell'epica— alle descrizioni. Il monito di moderare l'uso dell'ekphrasis prende di mira gli eccessi di alcuni storici, tipo quelli ridicolizzati da Luciano in Come si scrive la storia.

Parole chiave: ekphrasis, storia.

Abstract: As shown by numerous examples, historiography is the genre most congenial - even more than epic - to descriptions. The reminder of the rhetors to moderate use of ekphrasis takes aim at the excesses of some historians, such as those ridiculed by Lucian in How to write history.

Keywords: ekphrasis, historiography.
\end{abstract}

Recibido: 03-11-2014

Informado: 05-02-2015

Definitivo: 28-04-2015

La prima volta che compare, nella spuria Retorica di Dionigi di Alicarnasso ${ }^{1}$, il termine, l'ekphrasis già è presentata come un difetto, un errore, uno "sfoggio inutile e uno sciupio», una «lungaggine vana» di logoi.

Rh. 10,17 Alcuni hanno anche questo errore $(\dot{\alpha} \mu \alpha \dot{\rho} \rho \tau \mu \alpha)$, le cosiddette descrizioni ( $\alpha i$

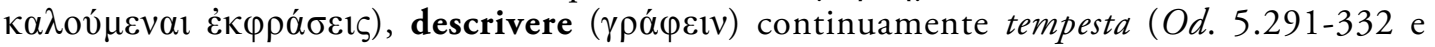
365-370, 10,47-55, Il. 14,392-397 e 398-401, A. Ag. 649-60 ecc.), pestilenze (Hom. Il. 1.61, Hes. Op. 243, A. Pers. 715, Th. 2,47,3), carestie (Hes. Th. 227 Limos personificato), schieramenti, aris-

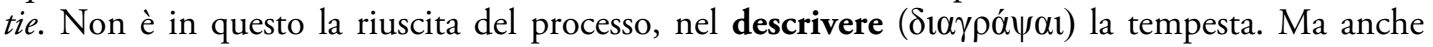
queste cose sono esibizione vana e spreco di discorso. Questo errore penetra nelle declamazioni per imitazione della storia e dei componimenti poetici. Ignoriamo, infatti, come sembra, che la sto-

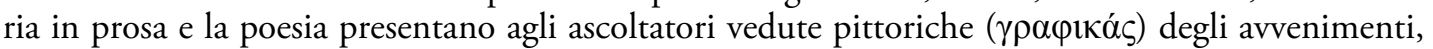

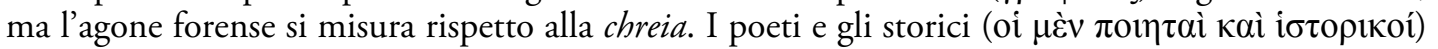

1 Cf. Berardi 2012, 190. Il termine si trova -in riferimento a descrizioni di topoi, pragmata e tropoi, cf. infra- anche in Imit. 31,3,2 e anche in questo caso è

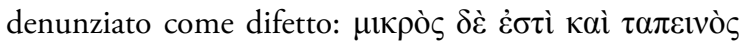

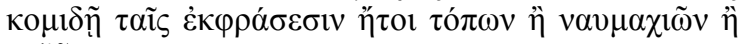
$\pi \varepsilon \zeta \tilde{\omega} v$. 
modellano gli avvenimenti con topoi e con prosopa, come sono avvenuti. I retori declamanti non avendo né un'idea condivisa né personale delle cose compiute, si plasmano vedute di pestilenze e di carestie e di tempeste e di guerre come essi dicono. È possibile dunque anche alla controparte descrivere ( $\varphi \rho \alpha ́ \sigma \alpha l)$ diversamente di come l'oppositore le dica. Come ho detto, dunque, anche queste cose sono una lungaggine vana di discorsi. Questo difetto è di uomini ignoranti, perché anche nei momenti decisivi degli agoni c'è una spinta idonea di fantasia ( $(\alpha \nu \tau \alpha \sigma i ́ \alpha \varsigma)$ e non c'è bisogno di srotolare dentro fantasie ( $\varphi \alpha \nu \tau \alpha \sigma i ́ \alpha \varsigma)$ dall'esterno con discorsi.

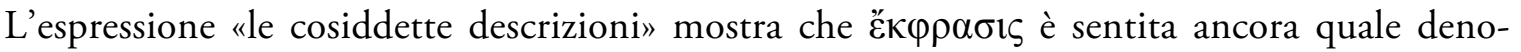

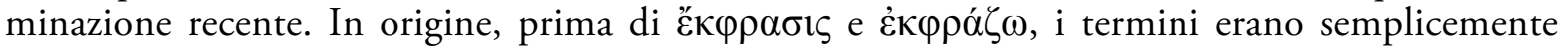

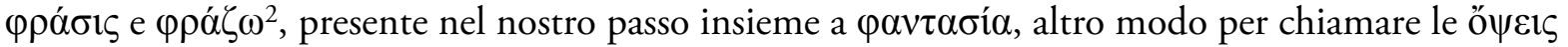

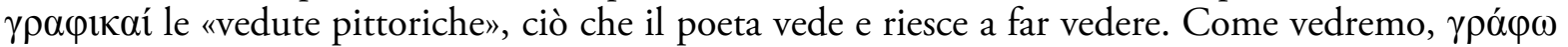
ha tra i suoi significati anche quello del futuro composto $\delta \alpha \gamma \rho \alpha ́ \varphi \omega$.

L'impressione di eccesso che l'ekphrasis dà dipende dall'accumulo dei dettagli, la cosiddetta $\dot{\varepsilon} \pi \varepsilon \xi \varepsilon \rho \gamma \alpha \sigma \alpha^{3} \alpha^{3}$, che però è proprio quella che assicura l' «alta definizione». Il difetto è conseguenza dell'«imitazione della storia e dei componimenti poetici», cioè, come ripete poco dopo, di «poeti e storici». Per i retori l'ekphrasis riguarda in primissimo luogo proprio la storia, benché proprio questo nesso non sia stato molto studiato ${ }^{4}$. Un problema più generale e di fondo è invece quello del rapporto tra "narrare» e «descrivere», sfiorato occasionalmente e, a mio parere, ancora del tutto sottovalutato ${ }^{5}$, perché è quasi impossibile narrare senza descrivere, senza fornire dettagli. Nicolao di Mira tenta di spiegare la differenza: "Differisce in questo dalla diegesi, che l'una passa in rassegna le cose generali, l'altra quelle particolari. Per esempio della diegesi è proprio dire: "combatterono gli Ateniesi e i Peloponnesiaci»; dell'ekphrasis che ciascuno di loro si servì di questa attrezzatura e di questo tipo di armatura» (Progimnasmi, Sull'ekphrasis, pp. 68,20-69,3 Felten). La differenza è tra "generale» e "particolare», ma di fatto consiste solo nella scelta e nel dosaggio dei dettagli, perché "Ateniesi e Peloponnesiaci» sono già dettagli, per quanto ancora generali. Va inoltre notato che l'esempio di Nicolao parafrasa il proemio delle Storie di Tucidide dove viene annunciato non solo il polemos ma anche "come» combatterono, $\omega \varsigma \xi \dot{\varepsilon} \pi \circ \lambda \dot{\varepsilon} \mu \eta \sigma \alpha v$ : "Tucidide di Atene $\xi v v \varepsilon ́ \gamma \rho \alpha \psi \varepsilon$ la guerra tra Peloponnesiaci e Ateniesi, come combatterono gli uni con gli altri».

Di storici e di storia Dionigi si intende, perché egli stesso è, oltre che un retore ${ }^{6}$, uno storico ed uno studioso di storici come mostrano le Antichità romane e la monografia Su Tucidide, nella quale spunta una critica contro la descrizione gratuita del lusso degli Ateniesi e dell'atletismo degli Spartani: «E che bisogno c'era, a proposito del lusso praticato anticamente dagli Ateniesi, di dire

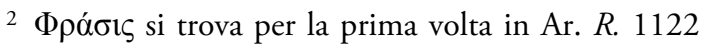
proprio in riferimento alla "descrizione» dei pragmata (uno degli oggetti dell'ekphrasis). Un esempio tardo di $\phi \rho \alpha ́ \sigma 1 \varsigma$ con l'antico valore di «descrizione» è nel titolo di un'orazione perduta di Dione, la Descrizione

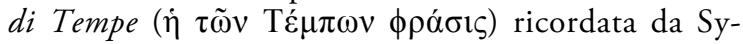

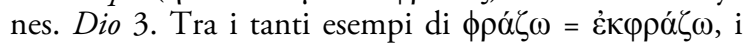

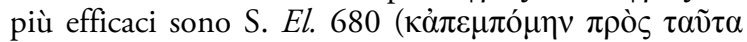

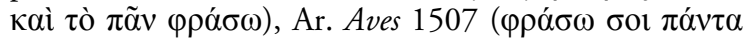

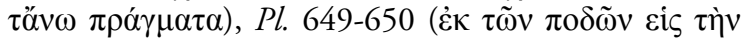

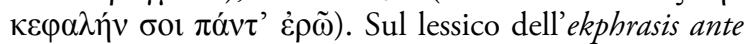
litteram tornerò in altra sede.

3 Cf. Schol. Od. $5.81 \delta i \alpha \grave{\eta} \tau_{\varsigma} \varepsilon \dot{\pi} \varepsilon \xi \varepsilon \rho \gamma \alpha \sigma i ́ \alpha \varsigma$ «ettagliando» e Nannini 1986, 55 n. 90, 62, 87-88.
}

4 Friedländer 1912 imposta la sua analisi per generi (epica, dramma, mimo, storia, epistolografia, oratoria); cf. inoltre Montanari 1984, Manieri 1998, 155-164 ( V. 'Evó $\rho \gamma \varepsilon 1 \alpha$ nella storiografia e nella pittura. 1. La storiografia») e Nicolai 2009, 34 e 41 (dove rinvia a Plin. Ep. 2,5,5 e 7,9,8).

5 Ravenna 1974 (negli scrittori latini), Fusillo 1985, 289-290, De Martino 2014 ${ }^{\text {bis. }}$.

6 Sui legami tra retorica e storiografia, dai quali dipende l'introduzione di orazioni nel discorso storico, cf. Manieri 1998, 155 e n. 519, che rinvia a Norden 1986. Su altri passi di Dionisio (epist. Pomp. 3,17,68 e Lys. 7,1,3) e su un altro grammatico-storico, Agatarchide di Cnido, cf. Berardi 2012, 68-69 e 58. 
$(\lambda \varepsilon ́ \gamma \varepsilon ı v)$ che intrecciavano i capelli sulla sommità del capo e portavano in testa delle cicale d'oro?» (Th. 1.6.3). O di dire che gli Spartani «furono i primi che si denudarono e che spogliatisi in pubblico si ungevano d'olio nel lottare» (19,856, p. 70,21-24 Pavano).

Poeti e storici. Dionigi usa il plurale per entrambe le categorie. Ma nei Progimnasmi i retori portano esempi di ekphrasis da un solo poeta, Omero ${ }^{7}$, spesso senza nemmeno nominarlo e trascurando tutti gli altri, inclusi i poeti tragici e comici autori di stupende descrizioni, veri e propri capolavori ecfrastici, non ancora adeguatamente studiati in quanto tali (Garzya 1997, De Martino 2013). Il plurale usato per gli «storici» era invece effettivo, perché nella scheda più completa, oltre che più antica, sull'ekphrasis, quella di Elio Teone, gli storici chiamati in causa sono più di uno: Erodoto, Tucidide, Ctesia, Filisto ${ }^{8}$.

Theo, Prog. X, pp. 66-69 Patillon-Bolognesi (= RhG I, pp. 118-120 Spengel):

L'ekphrasis è un logos circonstanziato9 che pone «sotto gli occhi» (i் $\pi$ 'ö $\psi \mathrm{lv}$ ) con evidenza

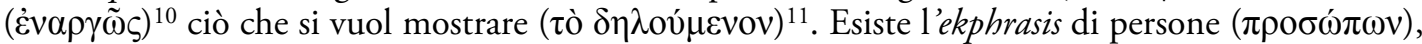

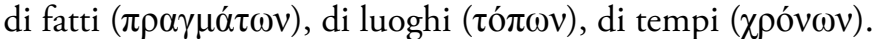

Di persone: per esempio il verso omerico Era curvo di spalle, scuro di pelle, crespo di capelli (Od. 19,246) e i versi relativi a Tersite era appuntito di testa, zoppo ad un piede e seguenti (Il. 2,219 e 217) e in Erodoto l'aspetto dell'ibis (2,76), degli ippopotami (2,71), dei coccodrilli egiziani $(2,68-70)$.

Di fatti: per esempio l'ekphrasis di guerra, di pace, di tempesta, di carestia, di pestilenza, di

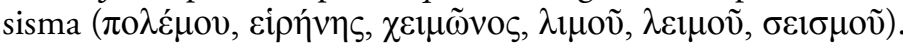

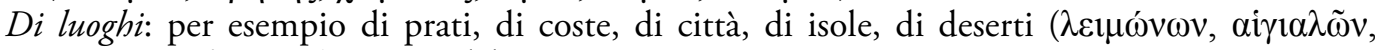

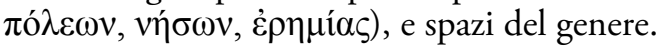

Di tempi: per esempio di primavera, di estate, di festa (

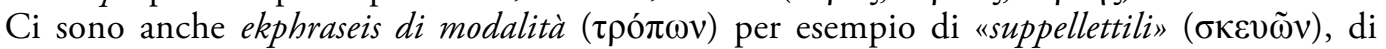
«armi« (ö $\pi \lambda \omega v)$, di «macchinari» ( $\mu \eta \chi \alpha v \eta \mu \alpha ́ \tau \omega v)$, «in che modo» ogni prodotto «è stato fabbricato» ( $\pi \alpha \rho \varepsilon \sigma \kappa \varepsilon v \alpha ́ \sigma \theta \eta)$, come in Omero la fabbricazione delle armi (Il. 18,468-616), in Tucidide la costruzione del muro d'assedio (ó $\pi \varepsilon \rho \imath \varepsilon \varepsilon \chi 1 \sigma \mu o ́ s)$ intorno a Platea $(3,21-22)$ e la fabbricazione dei macchinari ( $\dot{\eta} \tau \tilde{\omega} v \mu \eta \chi \alpha v \eta \mu \alpha \dot{\tau} \tau \omega v \kappa \alpha \tau \alpha \sigma \kappa \varepsilon v \eta \dot{)}$ «avendo diviso in due parti un grande tronco, lo incavarono tutto» (4,100). Nel libro 9 di Ctesia (FGrHist III, 688 F 9b Jacoby) per esempio «Vedendo le insegne dei Persiani su grandi tronchi all'alba verso l'acropoli in lontananza, i Lidi si volsero in fuga credendo che l'acropoli fosse piena di Persiani e già conquistata».

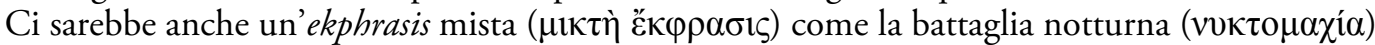
presso Tucidide $(2,2-4,7,43-44)$ e in Filisto (FGrHist $\mathrm{F} 52$ Jacoby $)^{12}$. La notte è infatti

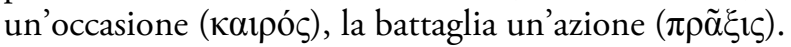

Questo esercizio ha una certa parentela con quello precedente: effettivamente entrambi non riguardano niente di definito, ma sono comuni e generali, per questo aspetto sono simili. Si differenziano invece in primo luogo in quanto il topos rientra fra le cose intenzionali, l'ekphrasis riguarda per la maggior parte cose inanimate e prive di scelta. In secondo luogo perché nel topos esponendo

\footnotetext{
7 Omero poteva tuttavia passare per cattivo storico, cf. Fornaro 2002.

8 Cf. Philist. T 20b Jacoby: «Bisogna deprecare l'incastrare in mezzo al racconto lunghe digressioni. Non bisogna semplicemente deprecare tutto il racconto come Filisto».

9 Il $\pi \varepsilon \rho$ í suggerisce l'idea che la descrizione è un circuito, un percorso, v. Dubel 1997.
}

10 Cf. Zanker 1961, Calame 1990, Manieri 1998. Su Teone, cf. anche Berardi 2012, 200.

11 Cf. Trifone, De tropis, RhG, III, p. 199,21-22 Spengel.

12 L'esempio dei retori è Th. 7,42-45 (il termine nyktomachia si trova solo appunto in Th. 7.44). Teone aggiunge Philist. Sulla nyktomachia di Triph. 506-691, cf. Miguélez Cavero 2007. 
i fatti aggiungiamo il nostro giudizio dicendo che sono o utili o perniciosi, mentre nell'ekphrasis l'esposizione dei pragmata è neutra.

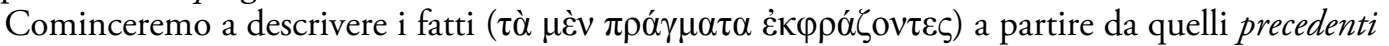
e da quelli concomitanti e da quelli conseguenti per esempio nel caso di una guerra passeremo in rassegna prima ( $\delta \varepsilon \varepsilon \xi \varepsilon \lambda \varepsilon v \sigma o ́ \mu \varepsilon \theta \alpha \pi \rho \tilde{\omega} \tau o v)$ i precedenti della guerra, il reclutamento dell'esercito,

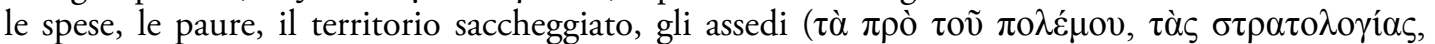

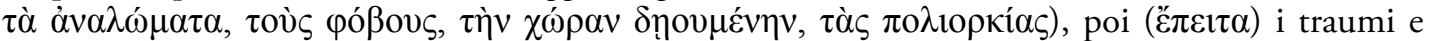

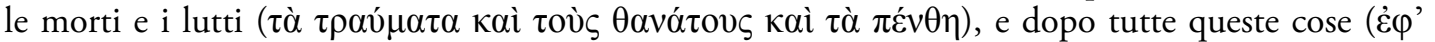

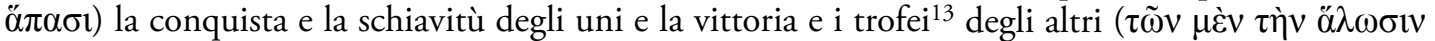

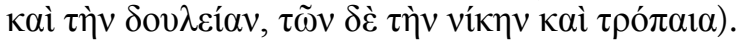

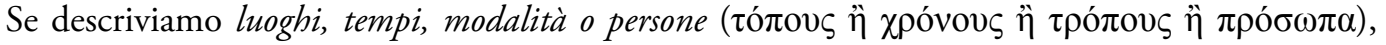
dopo la relativa esposizione ( $\mu \varepsilon \tau \grave{\alpha} \tau \tilde{\eta} \varsigma \pi \varepsilon \rho \grave{~} \alpha \hat{\tau} \tau \tilde{\omega} \nu \delta \eta \eta \eta ́ \sigma \varepsilon \omega \varsigma$ ), cominceremo a parlarne a partire

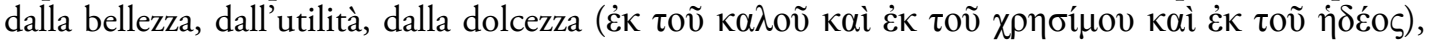

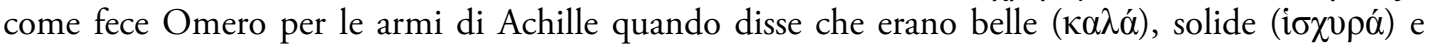

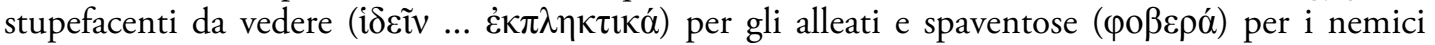

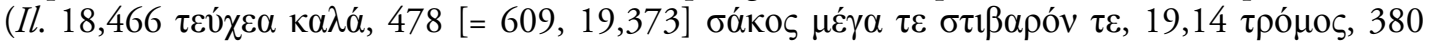

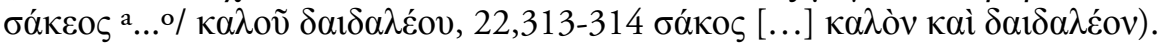

I pregi dell'ekphrasis sono i seguenti: chiarezza ( $\sigma \alpha \varphi \eta ́ v \varepsilon ı)$ soprattutto e evidenza di far quasi

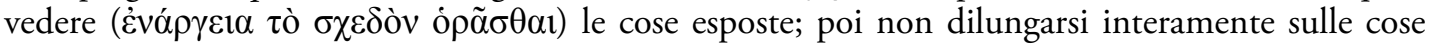

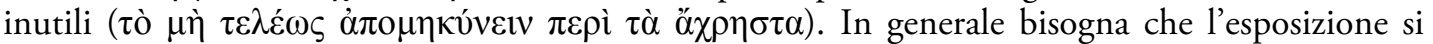

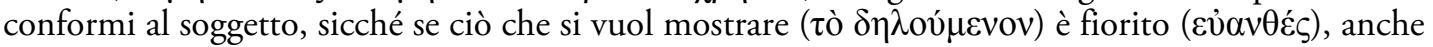

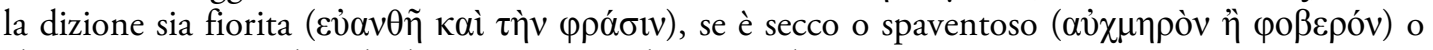
altrimenti, neppure lo stile deve stonare con la natura di quei soggetti.

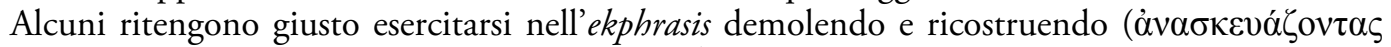

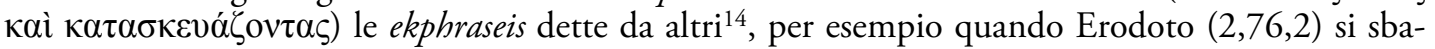
glia sull'aspetto dell'ibis dicendo che sono piuttosto bianchi di ali, tranne la testa, il collo e la punta della coda. Infatti la coda è tutta bianca. Ma a noi non sembrano dire nulla di nuovo rispetto alle cose già dette, perché riteniamo che questo genere di esercizi rientri fra le demolizioni e

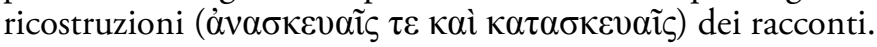

Fra gli altri retori che descrivono l'ekphrasis ${ }^{15}$ anche Pseudo-Ermogene e Aftonio fanno esplicito riferimento a Tucidide, lo 'Storico' per antonomasia:

Hermog., Prog. X. Sull'ekphrasis, pp. 202,2,5-10 Patillon: Di pragmata come la descrizione della pezomachia e della naumachia ${ }^{16}$. Di kairoi come pace, guerra. Di topoi come porti, litorali, città. Di chronoi come primavera, estate, festa. 3. Ci potrebbe essere anche una descrizione mista, come presso Tucidide $(7,44)$ la nyctomachia. La notte infatti è un kairos, la battaglia una praxis.

Aphth., Prog. XII, Definizione dell ekphrasis (pp. 147,1,6-148,2,7 Patillon: Pragmata come naumachie e pezomachie, come (fa) lo Storico. Kairoi come primavera e estate, descrivendo quali fra i fiori spuntano in esse ${ }^{17}$. Topoi come quando lo stesso Tucidide $(1,46,3-4$, cf. 1,30,3) disse

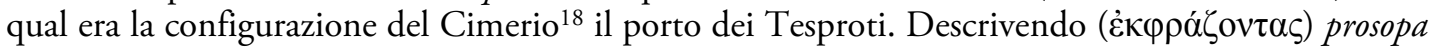

13 In E., Supp. 647 il Coro chiede al Messaggero "Come innalzarono il trofeo $(\tau \rho \circ \pi \tilde{i} \alpha)$ in onore di Zeus?». In Lisistrata 285 è ricordato il trofeo di Maratona, al v. 318 quello dei vecchi sulle donne.

14 Vid. Miguélez Cavero 2007, 500.

15 Trifone (RhG III, p. 201,27-31 Spengel) e Cocondrio ( $R h G$ III, p. 241,7-9 Spengel) descrivendo il charachterismos citano Od. 19,246 e Polibio di Sardiano (RhG III, 108,7-109,18) descrivendo l'eikonismos cita Od. 18,246 e Il. 2,219 e 217.

${ }_{16}$ Su pezomachia e naumachia come repertori grandiosi per scrittori e per pittori, cf. Demetr., Eloc. 75-76.

17 Per la fioritura, cf. Libanio, pp. 479-482 Foerster (specialmente 482,3-4). 
bisogna andare dalle prime alle ultime parti, cioè dalla testa ai piedi, (descrivendo) pragmata ama procedere dai precedenti a quelli concomitanti a quelli conseguenti, kairoi e topoi dagli esterni.

Delle ekphraseis alcune sono semplici, altre appaiate. E semplici come le pezomachie o naumachie dettagliate, appaiate come i pragmata e kairoi agganciati insieme, come quando Tucidide (7,43-44) descrive la nyktomachia in Sicilia. Riguardo alla battaglia precisava come fu fatta, riguardo alla notte com'era.

Aftonio ripete l'istruzione di Elio Teone di andare «dalla testa ai piedi» nel descrivere prosopa ${ }^{19}$. La stessa espressione si trova già in Aristofane (Pl. 649-650). La descrizione degli Ateniesi «che intrecciavano i capelli sulla sommità del capo e portavano in testa delle cicale d'oro» $($ Th. $1,6,3)$ seguiva dunque questa direzione dalla testa. Anche nella descrizione dei pragmata l'ordine è dall'inizio alla fine, cioè ugualmente dalla testa ai piedi.

La terminologia usata mostra che Teone sta pensando soprattutto alla storiografia. Tra i pragmata figura per primo polemos, che è il tema prioritario della storiografia, ma poi altri anch'essi

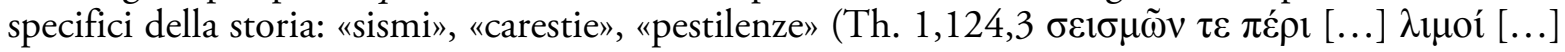

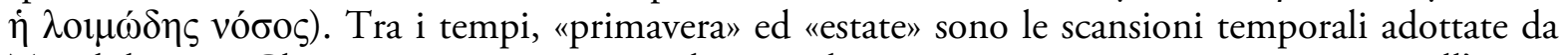
Tucidide: 2,1 «Gli avvenimenti sono stati descritti di seguito, come avvennero ciascuno nell'estate e nell'inverno" (cf. 3,116, 5,1 ecc.). Al concludersi di ogni inverno Tucidide aggiungeva la "firma». Se tra $\mathrm{i}$ «tempi» manca $\chi \varepsilon 1 \mu \omega ́ v$ è perché è già catalogato fra i pragmata col significato di «tempesta» (del resto tipicamente invernale), come quella descritta in A. Ag. 651-660, per la quale uno scolio

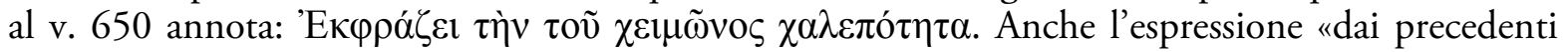

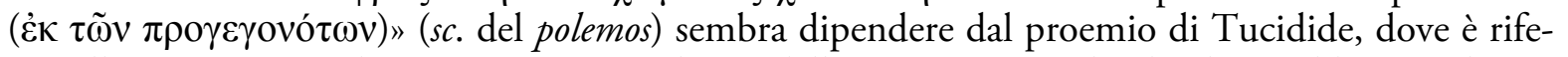
rita alle guerre "precedenti» non ai "precedenti» della guerra: "prevedendo che sarebbe stata la più grande e la più rilevante rispetto a quelle precedenti $(\tau \tilde{\omega} \nu \pi \rho 0 \gamma \varepsilon \gamma \varepsilon v \eta \mu \varepsilon ́ v \omega v) »(1,1,1 ;$ cf. $1,71,3 \tau \grave{\alpha}$ غ̇ं $\imath \gamma \imath \mu$ ó $\mu \varepsilon v \alpha$ «le novità»).

Erodoto. È una miniera di descrizioni, che meriterebbero di essere studiate tutte in quanto tali. Viaggiatore curioso ha descritto tutto ciò che di interessante vedeva. Famose sono soprattutto le descrizioni di beni culturali: opere d'arte (1,15, 50s., 2,124-125, 3,47, 60), edifici di Babilonia $^{20}(1,178 s s$.$) , piramidi (2,124,127,1$ e 136,3-4), Bubastis $(2,137)$, il labirinto $(2,148)$, il lago di Meride (2,149-150), il canale del Mar Rosso costruito ma solo parzialmente da Neco, il figlio di Psammetico $(2,158)$, il Ponto Eusino $(5,85)^{21}$. Famose sono anche le descrizioni di mezzi di trasporto, fra i quali le imbarcazioni del Nilo costruite con tavolette corte $(2,96,1-5)$ e le grandi zattere rotonde viste sull'Eufrate vicino a Babilonia $(1,194)^{22}$. Erodoto introduce quest'ultima ekphrasis usando il verbo $\varphi \rho \alpha ́ \zeta \omega$ che vale già come $\dot{\varepsilon} \kappa \varphi \rho \alpha ́ \zeta \omega$ :Comincio descrivendo ( $\varphi \rho \alpha ́ \sigma \omega v)$ la meraviglia più grande per me di tutte di quelle di qui, dopo la città». Un'altra ekphrasis particolare è quella delle tre tipologie diverse anche nel prezzo della mummificazione $(2,86-88)$, una vera e propria techne, che mostra tra l'altro l'alto livello di conoscenza dell'anatomia (Sierra Martín 2014).

18 Il porto Cimerio, situato fra due santuari, ha alle spalle l'omonimo promontorio Cimerio.

19 Cf. Nic., Prog. 69,12-17 Feltem e Berardi 2012, 196-197.

\footnotetext{
20 Cf. Ravn 1948.

21 Sulle descrizioni di Erodoto cf. Marasco 1978, 58-60, Casson 1978, 78-79.

22 Casson 1978, 75 e 83.
} 
Ma gli esempi che Teone prende da Erodoto sono di ben altro tipo, riguardano infatti descrizioni di animali ${ }^{23}$ (ippopotami e ibis) e provengono tutti dal libro II, quello dedicato all'Egitto.

Il retore ha scelto dunque come esempi dell'ekphrasis di prosopa le descrizioni di animali ${ }^{24}$ e le ha aggiunte con disinvoltura a quelle omeriche di esseri umani, Euribate e Tersite, come se ciò che contava fosse la singolarità dei prosopa. L'immissione di animali spalanca la categoria dei prosopa a tutti gli esseri anomali, da Scilla (Od. 12.85-100: 12 piedi, 6 colli, 6 teste, tre file di denti) a Gerione e Chimera tricefali (Hes. Th. $287 \tau \rho \iota \kappa \varepsilon ́ \varphi \alpha \lambda o v, 321 \tau \tilde{\eta} \varsigma \tilde{\eta} \nu \tau \rho \tilde{i} \varsigma \kappa \varepsilon \varphi \alpha \lambda \alpha i)$ ), a Cerbero con 50 teste (ivi, $312 \pi \varepsilon v \tau \eta \kappa o v \tau \alpha \kappa \varepsilon ́ \varphi \alpha \lambda o v)$ a tutti gli altri mostri.

La sezione erodotea sugli ippopotami ${ }^{25}$, è interessante anche per un altro aspetto. Infatti in

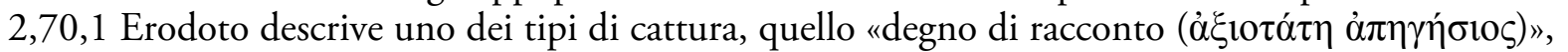
in realtà di descrizione. In questa occasione usa il verbo $\gamma \rho \alpha ́ \varphi \omega$ col significato che in futuro avrà $\delta 1 \alpha \gamma \rho \alpha ́ \varphi \omega$ «descrivo» (cf. infra).

L'ekphrasis dell'ibis a sua volta era comoda ad Elio Teone perché vi ritorna alla fine della sua scheda per segnalare che Erodoto aveva commesso degli sbagli, in particolare riguardo al colore di zampe, criniera, zanne, e coda ${ }^{26}$. Quello dell'ibis era un comodo esempio di ekphrasis da demolire e ricostruire, da sottoporre cioè ad anaskeue e kataskeue, i due momenti decisivi e proficui nel processo della ricreazione, del remake. Un bell'esempio di critica dell'ekphrasis della mischia di combattimento è in Tucidide 7,44, un paragrafo tutto dedicato a descrivere la peculiarità di una nyktomachia e la grande confusione inevitabile di notte. Tucidide osserva che persino di giorno in pieno combattimento è difficile identificare gli avversari: "Di giorno si conoscono i fatti più chiaramente, e tuttavia coloro che hanno preso parte a una battaglia, neppure questi li conoscono tutti, ma ciascuno sa solo e con difficoltà quel che è accaduto vicino a lui» (trad. G. Donini). Questa osservazione somiglia a quelle che Euripide mette in bocca a Eteocle nelle Fenicie 751-752 e a Teseo nelle Supplici 846-856 per criticare Eschilo che nei Sette a Tebe si attardava a descrivere dettagliatamente i sette assedianti e i loro scudi ${ }^{27}$.

L'ekphrasis dei coccodrilli e degli ippopotami è di animali veri, visti dal vivo ${ }^{28}$. Questo può spiegare perché Teone abbia ignorato la descrizione erodotea della mitica fenice ${ }^{29}$ che sta tra le due descrizioni erodotee di coccodrilli e ippopotami. Erodoto precisa infatti di aver visto la fenice solo in pittura. La sua è dunque una precoce descrizione di opera d'arte, cioè una tipologia che nei Progimnasmi verrà catalogata solo più tardi, da Nicolao di Mira: "Bisogna, quando descriviamo per caso soprattutto statue, dipinti o qualcosa del genere, cercare di aggiungere le motivazioni [...]» (Prog., X, RhG XI, p. 69,5-6 Felten).

23 Una curiosa descrizione è quella della riproduzione dei pesci Tilapia del Nilo $(2,93)$.

24 Altre descrizioni di animali sono per esempio in 3,102, 107ss., 4,183.

25 Essa ha come modello Ecateo (FGrHist I F 324). Eus. PE X 3, p. $466 \mathrm{~B}$ avverte infatti che Erodoto nel libro II trasferì letteralmente molte cose dalla Periegesi, adattando brevissime cose, quelle che riguardavano la fenicia $(2,73)$, l'ippopotamo $(2,71)$ e la caccia dei coccodrilli.

26 Erodoto descrive due tipi di ibis, quella crestata o eremita (Comatibis eremita) e l'ibis sacra (Ibis aethiopica), che è quella bianca, Lloyd 1989, 296. La pelle in- vece era usata per scudi e elmi secondo Plin. $H N$ 8,95, Lloyd 1989, pp. 290-291.

27 Cf. Méridier in Grégoire-Méridier-Chapouthier 2002, 135. Analoga polemica antieschilea è nella scena che precede il riconoscimento nell'Elettra di Euripide, cf. Quijada Sagredo 2013, 35 e nn. 8-9.

28 Una breve descrizione di coccodrilli e di ippopotami si troverà in futuro in Philostr. Im. 1,5 dove «i coccodrilli e gli ippopotami, che alcuni hanno dipinto insieme al Nilo, giacciono nel gorgo profondo, affinché i fanciulli non si spaventino" (Abbondanza 2009, 267 n. 22).

29 Anch'essa si basa su Hecat., FGrHist I F 324 Jacoby. Cf. van den Brock 1972. 
Tucidide. Per la descrizione della battaglia notturna, Teone porta come esempio Tucidide. In 2,2-4 è descritta la battaglia notturna (431 a.C.?) a Platea dei Plateesi contro i 300 Tebani che vi si erano infiltrati di notte, cioè la prima attività militare all'inizio della guerra del Peloponneso. In $7,44,2$ è descritto poi ciò che avvenne a Siracusa «in una battaglia notturna ( $\dot{\varepsilon} v \delta \dot{\varepsilon} v v \kappa \tau o \mu \alpha \chi i ́ a)$, l'unica che sia stata combattuta tra grossi eserciti nel corso di questa guerra".

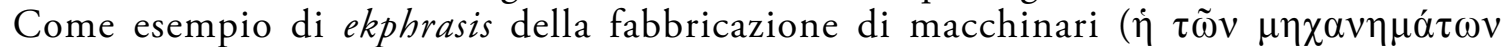

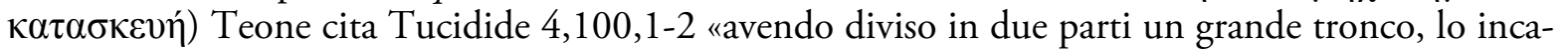
varono tutto».

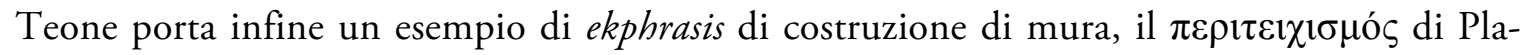
tea $^{30}$.

Le mura erano una realtà importante nella vita della polis, anche se si andava sostenendo che non erano tutto, e che le vere mura di una città erano gli andres ${ }^{31}$. La loro costruzione significava costruire o ricostruire una città. Nell'opuscolo Come si scrive la storia - sul quale torneremo- - Luciano suggeriva equilibrio nelle descrizioni di mura e di monti e fiumi: "Ci vuole il massimo equi-

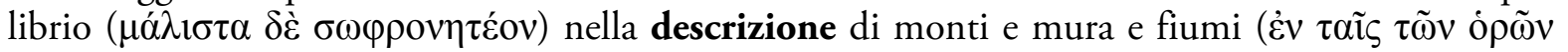

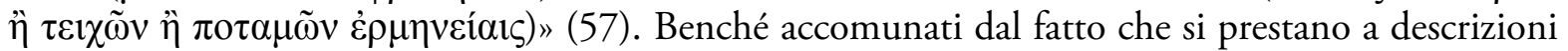
esagerate, monti e fiumi da un lato e mura dall'altro appartengono a tipi diversi di ekphrasis. Monti e fiumi rientrano in quella dei topoi, le mura in quella dei tropoi, perché ciò che viene descritto è la costruzione, come nel caso di armi e macchinari ${ }^{32}$.

L'esempio scelto da Teone è ancora una volta scialbo. Di alcuni celebri teichismoi (Troia, Tebe, Megara, Tirinto) abbiamo solo notizie indirette. Tra quelli le cui descrizioni sono sopravvissute i più interessanti sono infatti quelli di Babilonia in Erodoto, di Atene in Tucidide.

La descrizione delle mura di Babilonia e di Atene è consapevolmente di tropoi (Hdt. 1,179,1

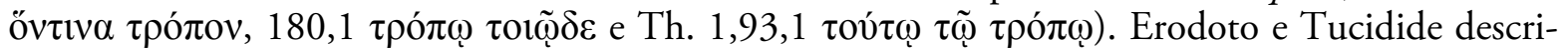
vono infatti il cantiere ${ }^{33}$, il processo attraverso il quale le mura vengono costruite. Interessante an-

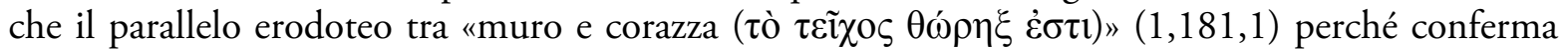
l'affinità delle mura ad armi e vestiti, tipologie in quanto oggetto dell'ekphrasis dei tropoi. In Ero-

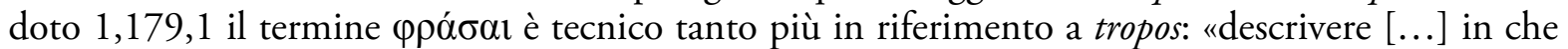
modo fu costruito il muro». Il verbo si trova già in Erodoto: 1,121,6 «E se vedessimo ( $\dot{\varepsilon} \omega \rho \tilde{\omega} \mu \varepsilon v)$

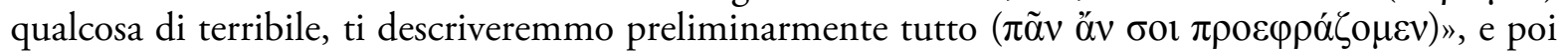

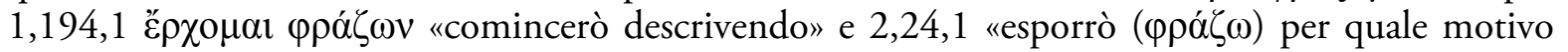
mi sembra che il Nilo si gonfi d'estate».

In Erodoto $(1,178,1-179,1$ e 3) la descrizione del teichismos si inserisce nel logos babilonese (177-200) che comincia con la descrizione della città. In 1,179,1 lo storico precisa che sta descrivendo il tropos: «Bisogna dire ( $\varphi \rho \alpha ́ \sigma \alpha \imath)$ inoltre dove fu impiegata la terra ricavata dal fossato e in

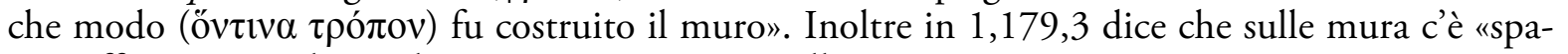
zio sufficiente per il giro di un carro a quattro cavalli».

30 Cf. Th. 3,20,3-21,2.

31 Alc. fr. 122 Voigt, Th. 7,77,7, Isocr. Pan. 13-14, Pl. Lg. 6,778d-770b, Arist. Pol. 1330b-1331a, Longo 1974 e 1975.

32 Il poema in 6500 versi attribuito a Epimenide Costruzione della nave Argo e viaggio in mare di Giasone verso la Colchide (D.L. 1,111) doveva consistere almeno nella prima parte proprio in una descrizione della costruzione e del cantiere navale. In D.L. 2,47 una Descri-

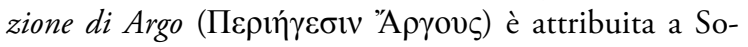
crate Storico.

33 Allo stesso modo in Hdt. 2,124,3-5 è descritto il cantiere della costruzione della piramide (Casson 1978, 78). 
Tucidide $^{34}$ parla invece delle mura del Pireo fatte erigere da Temistocle e fornisce alcuni dettagli. Il muro fu costruito in meno di un anno ${ }^{35}$, tra l'autunno 479 a.C. e la primavera 478 a.C. ${ }^{36}$, e collaborarono anche donne e bambini. Le mura erano così larghe che vi passavano due carri, non uno solo a quattro cavalli come nelle mura di Babilonia. Ancora maggiore la larghezza delle mura

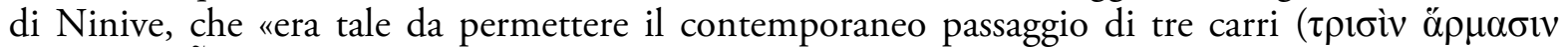

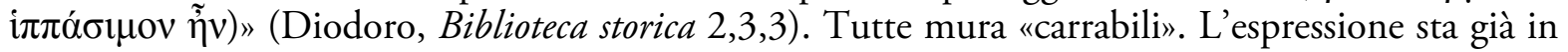

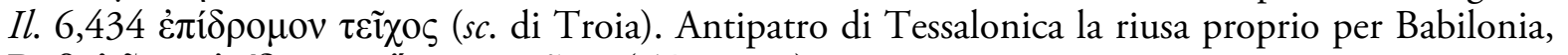

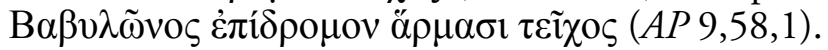

Ai due grandi teichismoi si rifa Aristofane negli Uccelli ${ }^{37}$. Il teichismos di Nubicuculia è una idea geniale di Pisetero, preannunciata ai vv. $550-552^{38}$. Egli dà poi istruzioni a Evelpide come aiutante-muratore (837-842) e successivamente domanda i dettagli tecnici al I Messaggero (1122-1169), che si cimenta in un'ekphrasis grandiosa e minuziosa come quelle tragiche ${ }^{39}$. Notevole anche la distinzione in opere murarie e opere in legno, tipica di cantiere navale, di un naupegos. Gli aspetti tecnici sono tantissimi. Vario è il personale sussidiario (841, 1161 guardiani; 842, 1160 ronda con la campanella), vari i materiali e gli attrezzi di lavoro: ghiaia ( $\left.\chi \dot{\alpha} \lambda \iota_{\kappa \alpha \varsigma}\right)$ (839),

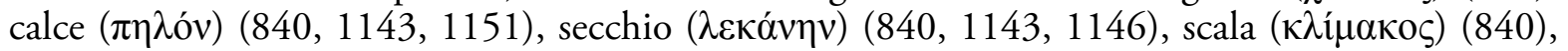

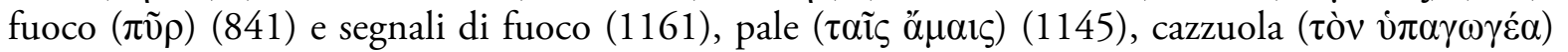

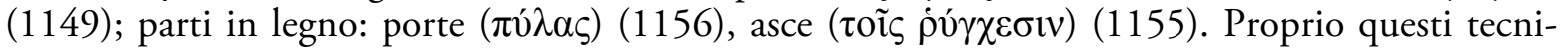
cismi fanno capire perché l'erezione delle mura sia definita dal I Messaggero un "prodotto bellis-

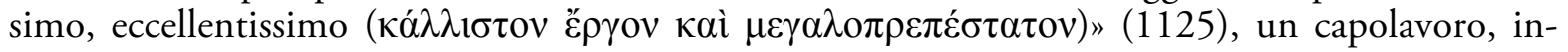
somma.

Quando Pisetero precisa che le mura della nuova città saranno «di grandi mattoni cotti: come Babilonia» (552), Aristofane esplicita la sua fonte (Hdt. 1,179). Anche il cenno al muro pelargico (832) o «delle cicogne», ai piedi dell'acropoli di Atene ${ }^{40}$, è ricordato in Erodoto 5,64,2 (cf. Arist. Ath. 19,5). Inoltre quando il I Messaggero fornisce l'altezza delle mura (100 braccia, cioè oltre 150 metri, anche se solo una metà di quanto Temistocle pensava) e afferma di averla misurata personalmente (1130) allude a Erodoto 2,127,1, dove lo storico dice di aver misurato personalmente la base della piramide di Chefren. La tecnica di misurazione dell'altezza delle mura, si basava sulle «file di mattoni» in una zona del muro non imbiancata ${ }^{41}$, ed è descritta in Tucidide 3,20,3-4, nel passo immediatamente precedente quello col teichismos (Th. 3,21) ricordato da Teone.

A questa tecnica fa riferimento anche Euripide nelle Fenicie 180-181 quando il Pedagogo dice che Capaneo «calcola ( $\varepsilon \varepsilon \kappa \mu \alpha i ́ p \varepsilon \tau \alpha)$ le scalate delle torri, misurando ( $\mu \varepsilon \tau \rho \tilde{\omega} v)$ le mura in alto e in basso».

34 Un altro strano doppio e simultaneo teichismos degli Ateniesi e dei Siracusani è descritto da Th. $6,99,1-100,3$.

35 Sui tempi di costruzione (anche autoriale), cf. De Martino 2004.

36 "In questo periodo gli Ateniesi cominciarono anche a costruire le lunghe mura fino al mare ( $\tau \grave{\alpha} \mu \alpha \kappa \rho \grave{\alpha}$

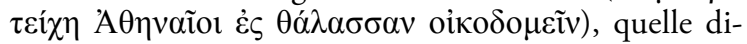
rette al Falero e quelle verso il Pireo» (Th. 107, 1). Sulle mura del Falero cf. 2,13,6.

37 Cf. Mastromarco 1977, Zanetto 1987, 270-271, Dunbar 1995, 595-597, 600, Totaro in MastromarcoTotaro 2006, 238-240 n. 246.
38 Il Corifeo accenna inoltre al muro pelasgico, cioè alle mura micenee dell'Acropoli (Hdt. 5,64,2, Arist. Ath. 19,5). Il muro era detto pelasgico perché fondato dai Pelasgi (Hecat., FGrHist 1 F 127, Myrsil. FGrHist 477 F 9, Paus. 1,28,3). L'espressione si riferisce anche alla zona sacra ai piedi dell'Acropoli.

39 L'epexergasia (scolio ad Od. 5. 81 "dia tes eperxergasias" "dettagliando») assicura l' "alta definizione».

40 Il riferimento è all' "assedio dei seguaci di Ippia entro il perimetro difensivo di Atene», cf. Nenci 1994, 251-252.

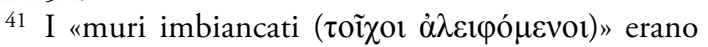
quelli spalmati di intonaco impermeabile. 
Altri dettagli presenti in Aristofane sono tucididei, anche se restano indirettamente anche erodotei $^{42}$. Erodoto 1,179,3 dice che sulle mura di Babilonia una quadriga può fare inversione di marcia. Aristofane invece parla di «due carri» che si incrociano (1126-1129). L'immagine della via a doppio senso, che permette il transito contemporaneo di due carri ciascuno in una propria corsia corrisponde a Tucidide 1,93,5 ed è competitiva rispetto a quella della via a senso unico alternato nella quale una quadriga può fare inversione. Ma il dettaglio del doppio senso di marcia sta già proprio in Erodoto 2,158 nell'ekphrasis del canale del Mar Rosso: "La sua lunghezza è di quattro giorni di navigazione; fu scavato in larghezza così che vi potessero navigare due triremi, fatte avan-

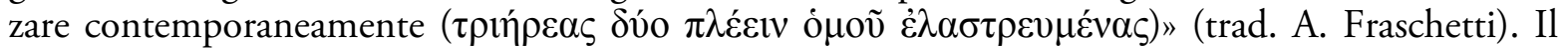
canale era largo poco meno di 31 metri, ma le triremi navigavano a soli $30 \mathrm{~km}$ circa all'ora, se facevano l'intero percorso in quattro giorni (Lloyd 1989, 375-376).

Il dettaglio dei carri — uno a quattro cavalli e due non sappiamo a quanti- che circolano sulle mura potrebbe essere alla base di alcune famose strutture architettoniche moderne come la porta di Brandeburgo (1793), l'Arc de Triomphe du Carrousel di Parigi (1815), il Politeama di Palermo (1890) o il Banco di Bilbao di Madrid e tante altre analoghe, caratterizzate dalla presenza di carri sulla sommità. Questi effetti dell'ekphrasis sull'arte anche moderna sono interessanti ${ }^{43}$.

Altri dettagli tucididei: le fondamenta sono in pietra e la parte superiore in legno (1,93,2 e vv. $1136,1139)$, la costruzione è frettolosa $(1,93,1-2$ e v. 1165) e collettiva e a cura di donne e bambini [1,90,3 (cf. D.S 1,40,1 e vv. 1148-1151)].

Dunque «le mura di Nubicuculia, erette con il fine di affermare l'egemonia della nuova città degli uccelli, si presentano come la proiezione celeste della mura che erano state erette da Temistocle più di mezzo secolo prima con il fine di affermare l'egemonia marittima di Atene» (Mastromarco in Mastromarco-Totaro 2006, 14). Ma «la descrizione tucididea della costruzione delle mura di Temistocle sembra condotta secondo il metodo erodoteo», "Tucidide «erodoteggia»" (Mastromarco 1977, 45).

Dopo Erodoto e Tucidide, Aristofane si impegna in un'ekphrasis di mura secondo i canoni per quanto ancora non scritti: dimensioni delle mura (altezza e larghezza), elenco analitico delle numerose maestranze (gru, cicogne, pivieri, uccelli, aironi, oche, anatre rondini), e soprattutto le quantità sia delle dimensioni che di alcune maestranze: trentamila gru (1136), diecimila cicogne (1139). Il «numero» sin dal catalogo omerico delle navi era un «dettaglio» essenziale delle descrizioni militari, anche se si era andato ridicolizzando per l'approssimazione e la faziosità esagerate ${ }^{44}$. Questi

42 Mastromarco 1977, Zanetto 1987, pp. 270-271, Dunbar 1995, 595-597, 600, Totaro in MastromarcoTotaro 2006, 238-240 n. 246.

43 Anche la dettagliata descrizione della corsa con le bighe, testimoniata nel discorso del falso Messaggero nell'Elettra di Sofocle, diventerà in futuro una scena tipica del cinema mitologico-storico, cf. De Martino $2014^{\text {bis }}$, 138-139.

44 Sul "numero» nelle descrizioni, cf. De Martino 2013, 214-216. La pignoleria nel numero segnala una descrizione seria o semiseria di un ergon speciale, cf. e.g. Saffo, fr. 109 Voigt, sulle scarpe del thyroros. Il numero dei contingenti anche navali è tipico dello storico, cf. e.g. Tucidide 1,27,2. Il numero dei caduti è un dato significativo storico-militare. Polibio 39,12 parla di «scontri e combattimenti in cui sono morti, magari, dieci soldati, o anche di meno, e ancora meno cavalieri [...] Costoro, quando si trovano a descrivere un assedio, sono costretti a riferire ogni progetto, ogni bravata individuale, ogni disposizione». Cf. Canfora 1974, 22. Ma i modelli sono poetici. Nei Persiani Eschilo precisa che i Greci avevano 300 navi circa e 10 navi scelte e Serse 1000, delle quali 207 erano veloci (339-342) e 250 erano di Taribi, comandante di "cinque volte/ cinquanta navi» (323). Nella hypothesis dei Persiani si precisa che "non si poteva contare il numero dei cavalli da lui guidati, mentre le navi erano 1207 o $1214 »$. 
numeri più adatti ad uno storico permettono anche a chi non è uno specialista di calcolare la grandiosità della costruzione e i tempi di realizzazione.

La costruzione di una città è un'occasione ideale per descrizioni, inclusa quella del progetto costruttivo (Uccelli 550ss.), richiesto dal Coro (548 «che bisogna fare?»), ma anche dell'arma "fai-date» la cui costruzione è descritta da Pisetero ai vv. 356-365: pentola, spiedo, salsiera o piatto, che nel loro insieme costituiscono un $\mu \eta \chi \alpha ́ v \eta \mu \alpha$, per usare il termine di Teone, o più semplicemente una $\mu \eta \chi \alpha v \eta$, che è il termine usato da Aristofane. Quando Evelpide dice a Pisetero "Ormai superi Nicia $\tau \alpha \tilde{i} \varsigma \mu \eta \chi \alpha v \alpha \tilde{\imath} \varsigma$ » (363) è da intendere verosimilmente nelle "attrezzature» militari ${ }^{45}$.

Abbiamo qui un bell'esempio di «demolizione» e «ricostruzione» di una ekphrasis. Anche Aristofane supera Erodoto e Tucidide. La sua novità è il teichismos a cura di animali, quasi da vecchia fattoria, entrando in competizione anche con Esopo, il favolista degli animali, chiamato esplicitamente in causa al v. 651 dove amathes "non istruito" è chi non conosce Esopo. Aristofane amava essere riconosciuto sophos $(N u$. 520). Conosceva Esopo, Erodoto, Tucidide e sapeva amalgamarli.

Luciano. Le schede dei retori non sono le uniche pagine specialistiche sull'ekphrasis. Una vera e propria monografia si può considerare infatti un opuscolo di Luciano degli anni 166-167, Come si scrive la storia, che è noto come «l'unico trattato esplicitamente teorico» (Canfora 1974, 3), "l'unico testo autonomo di teoria storiografica a noi giunto dall'antichità» (Manieri 1998, 155).

Tra poesia e storia e tra storia e encomio la differenza sarebbe proprio nella retorica ${ }^{46}$ e in particolare nell'esagerazione estranea alla storia, il cui unico intento è la verità ${ }^{47}$.

8. Questa gente, poi, sembra ignorare che altri sono i presupposti e le regole della poesia, altri della storia. In quella, la libertà è sfrenata e unica norma è ciò che piace al poeta [...] anche se vogliono esaltare Agamennone, nessuno impedirà loro di dire che, nel capo, è simile a Zeus, nel petto a suo fratello Posidone, nei fianchi ad Ares, e insomma il figlio di Atreo e di Aeropa dev'essere una sintesi di tutti gli dei, dato che non bastano da soli Zeus Posidone o Ares ad eguagliare la sua bellezza. Ma la storia, se adotta una tale piaggeria, cos'altro diventa se non una poesia pedestre, priva però di quella elevatezza espressiva, e ridotta perciò ad esibire tali stranezze senza il metro e perciò tanto urtanti? È un gran male, anzi grandissimo, se uno non sa distinguere il campo della storia da quello della poesia, ma introduce nella storia i ricercati ornamenti di quella, il mito, l'encomio e le connesse iperboli. (8; trad. L. Canfora, qui e in seguito)

L'esempio poetico di encomio è in realtà anche una descrizione di prosopon (Il. 2,478-607). Nei $\$ \$$ 14-32 Luciano passa in rassegna una genia di storici contemporanei ${ }^{48}$ della guerra partica di Lucio Vero (161-166), che aveva inserito troppe descrizioni e a sproposito, o degradando il modello, come nel caso di Crepereio Calpurniano di Pompeiopoli, imitatore di Tucidide, che aveva descritto la "peste» scoppiata a Nisibi, versione deteriore dell'ekphrasis della peste di Atene (2,47-53).

45 Per la possibilità che significhi «stratagemmi», cf. Totaro in Mastromarco-Totaro 2006, 155-156 n. 73.

46 Norden 1986 segnala i passi che invece testimoniano proprio il nesso tra retorica e storia: Cic. Brut. 42 , Orat. 66, Quint. Inst. Orat. 10,2,21, Plin. Epist. 5,8,9, Hermog. II,417,28 Spengel e appunto Luc. Hist. Cons. 7 (ma bisogna aggiungere anche 35, 51, 58, dove si accenna ai discorsi nei testi storici), cf. Manieri 1998, 155 n. 519 e Nicolai 2009, 34-35.
47 La verità senza retorica è adatta al pubblico scelto specifico del bravo storico: Th. 1,22,4, Plb. 8,1,6, Str. 1,1,23 e Montanari 1984, 16. Aristotele ipotizza che "anche se dessimo forma metrica all'opera di Erodoto, resterebbe nondimeno opera di storia" (Poetica 1451b), Canfora 1974, 5.

48 Raccolti nei FGrHist 203-210 di Jacoby potrebbero anche non essere mai esistiti, cf. Montanari 1984, 111. 
Il più sfrenato è uno storico anonimo, del quale Luciano (19-20) elenca minutamente i temi della descrizione di topoi (città, monti, pianure, fiumi, territori, antri), di armi (scudo) e vestiti ${ }^{49}$ (pan-

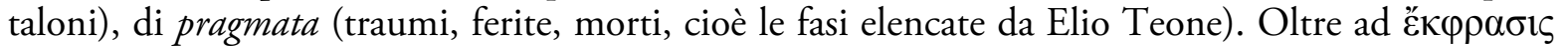
Luciano usa qui anche $\dot{\varepsilon} \rho \mu \eta v \varepsilon v ́ \omega$ col senso di $\dot{\varepsilon} \kappa \varphi \rho \alpha ́ \zeta \omega$ (cf. $27 \dot{\varepsilon} \rho \mu \eta v \varepsilon v ́ o v \sigma ı v$ ) e $\sigma v \gamma \gamma \rho \alpha ́ \varphi \omega$

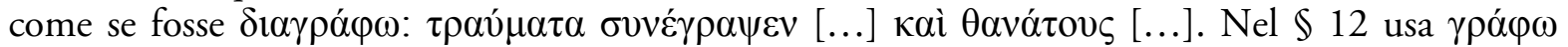
( $\gamma \rho \alpha ́ \psi \alpha \nu \tau o \zeta$ «descrivendo", cf. infra) che è già in Dionigi di Alicarnasso (cf. supra) e nel $\$ 57$

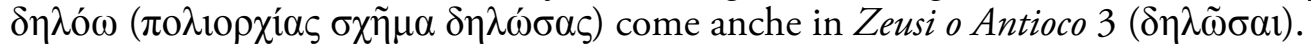

Tra tutti questi termini il più interessante è $\sigma v \gamma \gamma \rho \alpha ́ \varphi \omega$, usato proprio per lo scudo di Achille nel romanzo Alessandro il Macedone $(42,9 / 11)$. Sia $\gamma \rho \alpha ́ \varphi \omega$ che $\sigma v \gamma \gamma \rho \alpha ́ \varphi \omega$ figurano in passi programmatici di storici, dove potrebbe significare «de-scrivo», prima ancora o oltre che "scrivo",

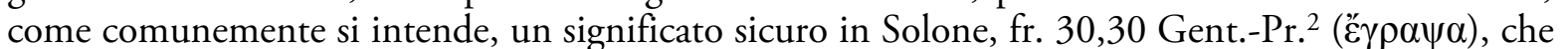
si vantava di aver usato un nuovo medium rispetto a legislatori orali. Ma negli storici potrebbe invece prevalere nel verbo il significato di «descrivere», più adatto al racconto storico.

Erodoto 2,70,1 usa $\gamma \rho \alpha ́ \varphi \omega$ per l'ekphrasis della cattura degli ippopotami: "I modi di catturarli (sc. gli ippopotami) sono molti e svariati, descrivo quello ( $\tau \alpha v ́ \tau \eta v ~ \gamma \rho \alpha ́ \varphi \omega)$ che mi sembra più

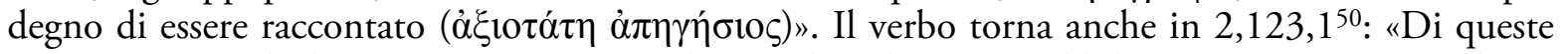
cose raccontate dagli Egiziani se ne serva colui per il quale sono credibili. Ma per me in tutto il rac-

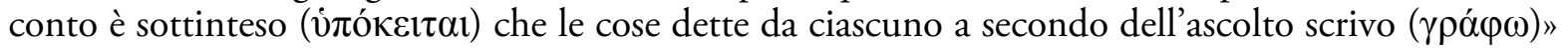

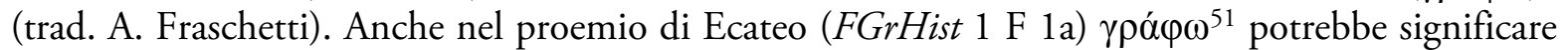
"de-scrivo": "Ecateo di Mileto così comunica: queste cose scrivo ( $\tau \alpha ́ \delta \varepsilon \varepsilon \gamma \rho \alpha ́ \varphi \omega)$ come a me sembra che siano vere. I logoi dei Greci sono molti e ridicoli, come a me paiono».

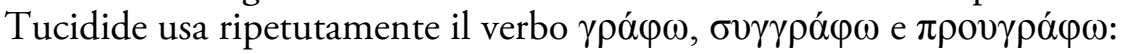

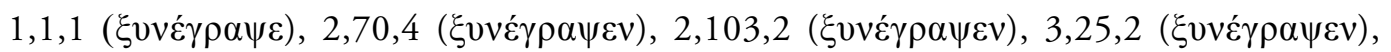
3,88,4, 3,116,3, 4,51, 4,104,4, 4,135,2, 6,7,4, 6,93,4, 7,18,4, 8,6,3, 8,60,3. 1,22,2 «Le imprese fra quelle fatte nella guerra non apprendendole da chi capitava ho deciso di descrivere $(\dot{\eta} \xi \dot{i} \omega \sigma \alpha$

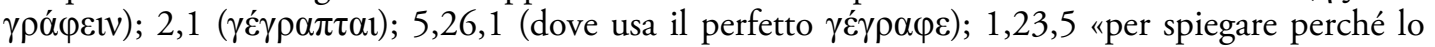
ruppero (sc. il trattato dei trenta anni) ho descritto prima $(\pi \rho 0 v ́ \gamma \rho \alpha \psi \alpha)$ le cause e i disaccordi»; cf. Hes., Op. 655 «molti descritti prima $(\pi \rho 0 \pi \varepsilon \varphi \rho \alpha \delta \mu \varepsilon ́ v \alpha) /$ premi avevano posto del magnanimo i figli», Hdt. 1,121,6 «E se vedessimo $(\dot{\varepsilon} \omega \rho \tilde{\omega} \mu \varepsilon v)$ qualcosa di terribile, ti descriveremmo preliminar-

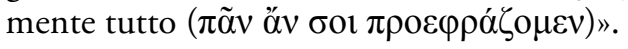

Pausania 2,17,4 duplica la formula:

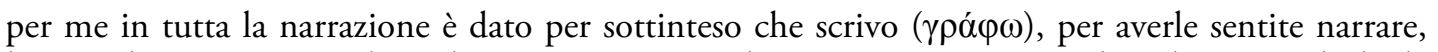
le cose che mi vengono dette da ciascuno. Questo logos e quante cose simili si dicono sugli dei le scrivo senza accoglierle, ma nondimeno le de-scrivo ( $\gamma \rho \alpha ́ \varphi \omega, \gamma \rho \alpha ́ \varphi \omega \delta \varepsilon ́)$.

Mentre Dionigi definiva la descrizione uno «sciupio», una «lungaggine vana di discorso», Luciano usa invece la metafora alimentare e tradizionale: chi descrive troppo è come un cafone che

49 Sull'abbigliamento già efficamente descritto in Omero, cf. De Martino 2012. Una costruzione delle corazze sarmatiche con zoccoli di cavalli e nervi di cavalli e di buoi si trova in Paus. 1,21,6.

50 Il criterio è ripreso in 7,152,3 «Quanto a me sono obbligato a riferire dettagliatamente ciò che è stato detto, ma non sono obbligato a credere ogni cosa, e questo principio può essere ritenuto valido per me in tutta la mia narrazione».

51 Cf. De Martino 2008, 64-68 («Scrivere storia»), e $2013^{\text {bis }}, 158$, dove va aggiunto il $\gamma \rho \alpha ́ \varphi \omega$ di Erodoto. Segnalo qui anche A. Supp. 179 (metafora delle tavolette della mente usata da Danao per le Danaidi). 
(safficamente) «non ha mai saputo come si indossa un abito» e che di fronte ad una tavola ric-

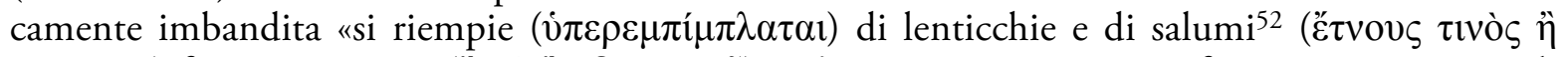

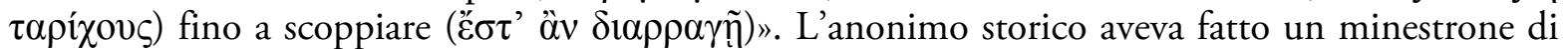
descrizioni, dallo scudo dell'imperatore, ai pantaloni di Vologene, alla chioma di Osroe, ai ferimenti ma in zone non vitali del corpo, come l'«alluce», verosimilmente variante inedita e grottesca del «tallone» di Achille ${ }^{53}$, e di morti molteplici per un semplice urlo del governatore Prisco.

L'eccesso è nella quantità, nel dosaggio ed anche nella scelta di dettagli inverosimili, fra i quali in primissimo luogo quelli numerici.

Lo storico preso in giro è anonimo. Il suo limite è che descrive "per ignoranza di ciò che va nar-

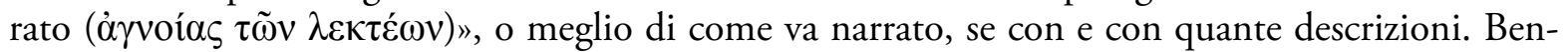
ché anonimo, lo storico è presentato all'inizio «simile a Tucidide, o forse anche un po' migliore». Le frecciate contro questo stra-Tucidide sono dunque anche contro il vero Tucidide, anche lui descrittore.

19. Ce n'era poi un altro, notevole per l'eloquenza, anche lui simile a Tucidide, o forse anche un po' migliore, il quale si era preso la briga di descrivere (i $\rho \mu \eta v \varepsilon v ́ \sigma \alpha \varsigma)$ con la massima chiarezza

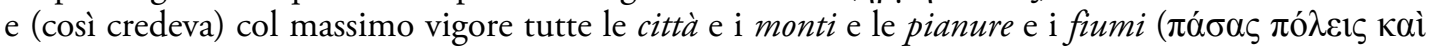

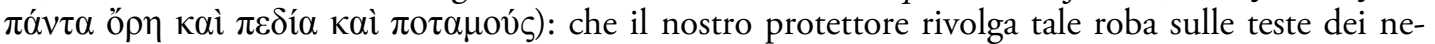
mici, tale era il gelo di quello scritto, peggio della neve del Caspio o del ghiaccio celtico! Per esempio, a stento gli bastava un intero libro per descrivere ( $\dot{\varepsilon} \xi \eta \rho \mu \eta v \varepsilon v ́ \theta \eta)$ lo scudo dell'imperatore, con la Gorgone al centro, i cui occhi sono colorati di azzurro, bianco e nero, la cintura color dell'iride e i capelli serpenti intrecciati come riccioli. Poi i pantaloni ( $\dot{\alpha} v \alpha \xi v \rho i ́ s)$ di Vologese e le briglie (ó

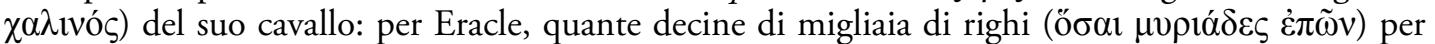

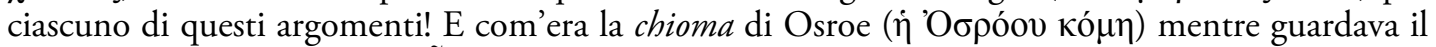
Tigri e in quale caverna ( $\dot{\varepsilon} \zeta$ oĩov öv $\tau \rho \circ v)$ si rifugiò, ombreggiata di edera, mirto e alloro lussureggianti tutt'intorno! E considera un po' come tutto questo sia necessario alla storia e tale che senza, non sapremmo per bene come andarono le cose.

20. In realtà, è per incapacità di raccontare quello che sarebbe necessario, o per ignoranza di ciò che merita di essere narrato che costoro ricorrono a tali mastodontiche descrizioni di personaggi e

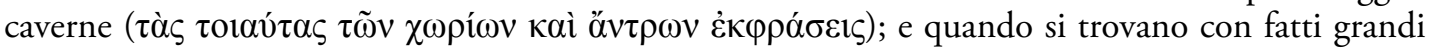
e molteplici sembrano schiavi da poco arricchiti, che hanno appena ereditato le sostanze del padrone: gente che non ha mai saputo come si indossa un abito o come si sta a tavola e che, dinanzi

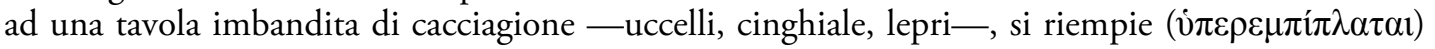

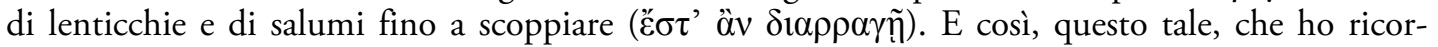

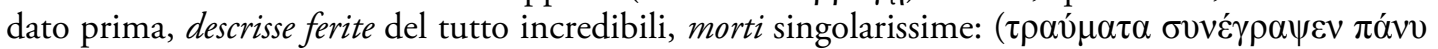

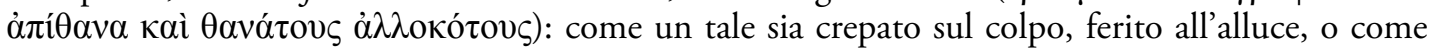
ad un semplice urlo del governatore Prisco siano morti ventisette nemici. Persino per quel che ri-

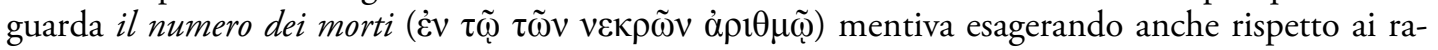
pporti di comandanti: sosteneva che presso Europo fossero morti 70.236 nemici e da parte romana

52 In Rane 62-67 la poesia di Euripide è paragonata a un passato di legumi (Pellegrino 2000, 12 n. 11). Il passato di lenticchie ritorna in un episodio della vita di Zenone in Diogene Laerzio VII 3. Cratete chiede a Zenone di portare una pentola di lenticchie attraversando il Ceramico, ma Zenone si imbarazza e cerca di nasconderla. Cratete gliela rompe e il passato gli cola addosso. Sul bollito di fave, cf. Timone, fr. 3 Di Marco. La metafora alimentare è usata da Luciano anche in 44 «l'opera non deve essere adorna di figure troppo turgide o scopertamente ricercate, altrimenti il risultato rassomiglierà ad un cibo troppo condito» e 56 «Neanche [...] nel bel mezzo dei manicaretti —uccelli, lepri, cinghiali selvatici, ventresche - imbandirai anche aringhe e lenticchie sol perché anche questo è pronto; tutt'altro; i cibi grossolani li lascerai da parte».

53 Un esempio omerico di ferimento falsamente mortale è quello di Menelao in Il. 4,127-219. 
solo due e nove feriti. E non so proprio chi, se non un pazzo, potrebbe tollerare cifre del genere. (trad. L. Canfora)

Sull'ekphrasis Luciano torna anche nei $\$ \$ 12$ e $57^{54}$. Nel $\$ 12$ ricorda la lettura di uno storico di fronte ad Alessandro bruscamente interrotta ${ }^{55}$ proprio dal re che buttò nel fiume il libro perché esageratamente falso. Il brano che lo storico leggeva era quello che era solito leggere, un pezzo da repertorio, perché evidentemente lo considerava il suo pezzo migliore.

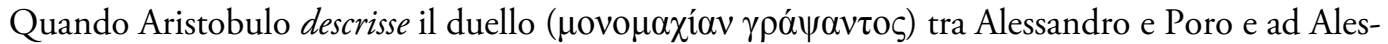

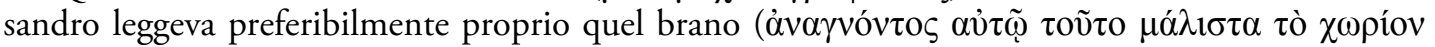
$\tau \tilde{\eta} \varsigma \gamma \rho \alpha \varphi \tilde{\eta} \varsigma$ ) (pensava di ingraziarsi al massimo il sovrano inventandogli false gesta ed imprese superiori al vero), Alessandro afferrò il libro e, dato che navigavano sul fiume Idaspe, lo gettò a capofitto nell'acqua, dicendo: «Anche tu, Aristobulo, dovresti fare questa stessa fine, tu che affronti duelli al posto mio e sgomini elefanti con una sola freccia!».

L'episodio che lo storico amava leggere era l'ekphrasis del duello, come mostrano i termini ( $\gamma \rho \alpha ́ \psi \alpha \nu \tau o \zeta$ e $\tau \tilde{\eta} \varsigma$ $\gamma \rho \alpha \varphi \tilde{\eta} \varsigma)$ usati da Luciano e che di nuovo ne confermano l'uso al posto dei più

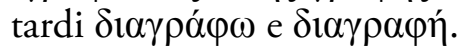

Nel $\$ 57$ discute la descrizione di «monti e mura e fiumi», come casi tipici di esagerazione da parte di cattivi storici che «lasciata da parte la storia» sfoggiano la bravura e il proprio "fare» (il proprio savoir faire) e non riescono a contenersi e a passare oltre, come invece sanno fare Tucidide e persino Omero, che in quanto poeta e non storico era libero di eccedere, ma che si conferma campione assoluto di moderazione rispetto a poeti come Partenio, Euforione, Callimaco. Nel caso di Tucidide Luciano porta esempi specifici di descrizioni moderate: un mechanema, lo schema dell'assedio e lo schema delle Epipoli o il "porto di Siracusa» ${ }^{56}(7,43-44)$, cioè il muro fatto costruire da Gilippo sulle Epipoli a ovest di Siracusa per impedire il completamento del muro che gli Ateniesi stavano costruendo a nord della città. Luciano giustifica persino la descrizione più vistosamente ampia, quella della peste di Atene, mostrando che

57. Ci vuole il massimo equilibrio nella descrizione di monti e mura e fiumi. Il fine è di esibire goffamente le proprie capacità rettoriche e di mettersi in mostra trascurando il racconto, ma, al contrario, dopo un cenno necessario per la chiarezza, procedere oltre evitando la trappola e questo genere di attrazioni; come fa Omero nella sua saggezza: pur essendo un poeta lascia perdere Tantalo, Issione, Titio e compagnia; ma se fosse Partenio, o Euforione, o Callimaco a parlare di questi episodi, quanti versi pensi che avrebbero impiegato per far scendere l'acqua alle labbra di Tantalo o per mettere Issione alla ruota? Guarda piuttosto Tucidide quanto di rado ricorre a questo genere

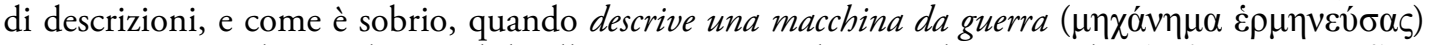

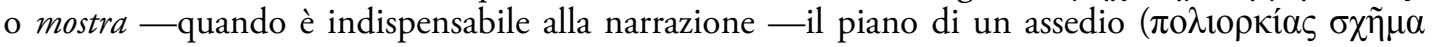

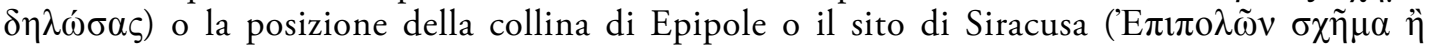

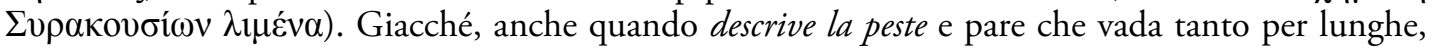

54 Sui $\$ 56-57$, cf. Avenarius 1956, 142ss., Montanari 1984, 116.

55 Un'analoga interruzione da parte del cinico Demetrio è testimoniata dallo stesso Luciano per un discorso da messaggero durante una lettura a Corinto «al punto in cui il messaggero racconta la sventura di Penteo e l'orrenda azione di Agave» (Ind. 19).
56 Il termine mechanema figurava nella scheda di Te-

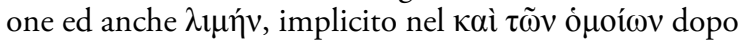
l'elenco dei topoi (prati, costiere, città, isole, deserti), è esplicito in Hermog. Prog. X. Sull'ekphrasis, p. 202, 2,6-7 Patillon «Di topoi come porti, litorali, città» e in Nicol. (Prog. X, RhG XI, pp. 68,14-15 Felten) «Topoi, per esempio prati, porti, laghi, e quante cose simili». 
considera la gravità dell'episodio e vedrai che anche in quel caso la descrizione è spedita e come abbraccia quasi di passata una folla di particolari.

Luciano (39) ricorda anche Ctesia contrapponendolo a Senofonte. Ctesia è medico personale di Artaserse, ma proprio per questo è condannato ad essere anche storico personale. Gli «elogi profusi $(\tau \tilde{\omega} \nu \dot{\varepsilon} v \tau \tilde{\eta} \gamma \rho \alpha \varphi \tilde{\eta})$ » saranno consistiti spesso proprio in descrizioni esagerate e cortigiane. Il termine $\gamma \rho \alpha \varphi \eta$ indica qui lo «scritto", ma come abbiamo suggerito, una storia è inevitabilmente $\gamma \rho \alpha \varphi \eta ́$ in entrambi i sensi. Anche Senofonte e Tucidide non possono che de-scrivere, ovviamente con molta più misura e bravura di questi storici decadenti.

Lo storico ha un solo compito: di dire come effettivamente sono andate le cose. Ma questo non potrà farlo finché avrà timore di Artaserse, essendo suo medico personale, o finché coltiverà la speranza di ottenere, in cambio degli elogi profusi nella sua opera, un mantello di porpora o una collana d'oro o un cavallo di Nisea. Ma questo non lo farà Senofonte, storico incorruttibile, né Tucidide.

Le descrizioni criticate da Luciano dovevano essere davvero stucchevoli. Anzi sarebbe interessante esaminare la Storia vera per verificare se e come le usa in quell'opera para-storica. Egli è infatti un esperto di ekphrasis e dei vantaggi. Ai $\$ \$ 49-50$ suggerisce di usare la «visuale comune»

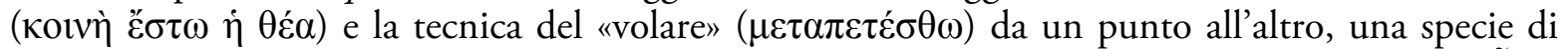
panoramica cinematografica, di visuale super o forse meglio apud partes che permette di cĩval

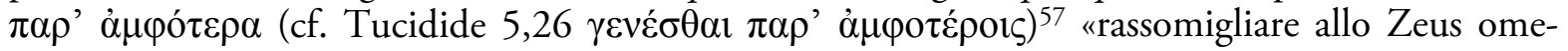
rico» (49) che ora guarda una terra ora un'altra e osserva e descrive gli avvenimenti «dall'alto», una tecnica usata oggi nel cinema, e di tenere d'occhio i comandanti e gli inseguimenti ${ }^{5}$ e le ritirate. Questo criterio, guardare dall'alto è lo stesso esplicitato nel $\$ 27$, quando critica quelli che trascu-

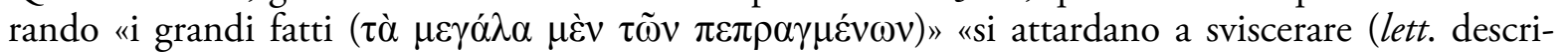
vono al rallentatore) i più piccoli con molta insistenza e diligenza ( $\tau \dot{\alpha} \mu 1 \kappa \rho o ́ \tau \alpha \tau \alpha \pi \alpha ́ v v$ $\lambda ı \pi \alpha \rho \tilde{\varsigma}$

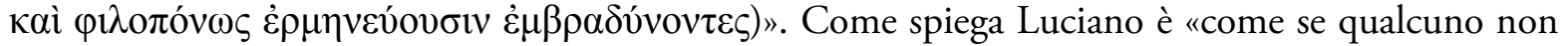

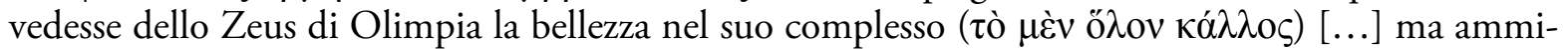
rasse la rifinitura e la levigatezza dello sgabello, la linea armonica della base spiegando con molta

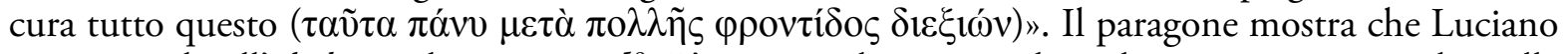
sta pensando all'ekphrasis di una statua ${ }^{59}$. L'esempio che porta subito dopo è uno storico che nella sua lettura dedicò sette righe alla battaglia di Europo e "venti misure d'acqua» all'incontro marginale tra un cavaliere mauritano (Mausaca) con un contadino, incluso il menu della cena, la flautista fatta arrivare e i doni scambiati fra i quali una "fibbia», altro tipico oggetto da descrizione.

Luciano stesso esegue molte descrizioni ${ }^{60}$ e così efficaci da essere tradotte secoli dopo in opere reali. Quella della Calunnia di Apelle in Non bisogna prestar fede alla Calunnia ha ispirato una tavola di Sandro Botticelli, conservata a Firenze nella Galleria degli Uffizi (Lovisetto 2005). Il quadro Alessandro e Rossane descritto in Erodoto o Aezione ha ispirato una sanguigna di Raffaello conservata all'Albertina di Vienna. Una monografia tutta di descrizioni è La Sala e anche Come si scrive la storia è un pot-pourri di descrizioni e si apre simbolicamente con una stupenda ekphrasis

7 Canfora 1974, 25.

58 Sull'«inseguimento», cf. Arist. Po. 1460a.
59 In Icaromenippo 17 Menippo dice che dall'alto le cose si vedono come i fatti sullo scudo di Achille (Il. 18,468-615).

60 Per una raccolta, cf. Maffei 1994. 
di una epidemia, uno dei temi dell'ekphrasis dei «tempi». L'epidemia è quella paradossale scoppiata ad Abdera, l'infatuazione per la tragedia e in particolare per quella di Euripide. Luciano parla come alcuni servi di Aristofane quando descrivono le malattie soprattutto mentali dei loro padroni ${ }^{61}$, solo che qui sono descritti i sintomi di un popolo intero, un'epidemia appunto: febbre (sei giorni), emorragia nasale (settimo giorno), sudore e mania per la tragedia (Andromeda e Perseo con la Medusa in testa). Il contagio sarebbe stato in seguito ad una messinscena estiva, cioè nella stagione sbagliata, dell'Andromeda da parte dell'attore Archelao. Ad essa somiglia quella scoppiata dopo la sconfitta di Elegeia nel 162 d.C., ossia l'infatuazione per la storia: «non c'è nessuno che non componga storie, o meglio sono diventati tutti altrettanti Tucididi ed Erodoti e Senofonti» (2). Spunta qui uno storico, che mancava nelle sezioni sull'ekphrasis dei Progimnasmi, ma che ha anche lui alcune descrizioni sia pure rapide, quasi in forma di appunti ${ }^{62}$, come quella mista all'inizio di Anabasi 1,5. Inizia come ekphrasis di topoi (un pezzo del deserto d'Arabia) e poi si trasforma in descrizione di prosopa, anche in questo caso di animali, soprattutto asini selvatici, e di tropoi, modi di catturarli.

Plutarco. Due testimonianze su Duride cattivo storico perché fa cattive descrizioni si trovano in Plutarco, Alcibiade 32 e Pericle $28^{63}$. In entrambi i casi è l'eccesso di dettagli descrittivi, assenti negli altri storici, che indiziano Duride come cattivo storico che inventa particolari suggestivi ma falsi.

Pl. Alc. 32 Duride di Samo (FGrHist 76 F 76) però, che dice di essere un discendente di Alcibiade, aggiunge a questo altri elementi, cioè che Crisogono, già vincitore ai giochi Pitici, suonando col flauto dava il ritmo ai rematori, e i comandi erano impartiti dall'attore tragico Callippide; e costoro indossavano tuniche e lunghe vesti e il resto dell'ornamento teatrale; e la nave ammiraglia entrò nel porto con una vela purpurea, come se facessero baldoria uscendo da un abbondante festino. Ma né Teopompo né Eforo né Senofonte scrivono queste cose, e non è credibile che Alcibiade lussureggiasse così arrogantemente tornando fra gli Ateniesi dopo un esilio e tante sventure: sembra invece che approdò timorosamente... (trad. Montanari 1984)

Per. 28 Duride di Samo (FGrHist 76 F 67) a questo aggiunge elementi da tragedia, accusando gli Ateniesi e Pericle di grande crudeltà, cosa che non si trova nelle opere né di Tucidide né di Eforo né di Aristotele. Ma non sembra che dica la verità, per esempio che Pericle fece condurre nell'agorà di Mileto i capitani e gli equipaggi dei Sami e li tenne legati a delle tavole per dieci giorni, e quando poi furono ridotti a malpartito ordinò di finirli a bastonate sulla testa e gettare i corpi senza sepoltura. Duride invero, anche quando non è personalmente coinvolto, per sua abitudine non riesce a mantenere il racconto entro i limiti della verità e ancor di più in questo caso sembra aver ingrandito le disgrazie della sua patria per calunniare gli Ateniesi. (trad. Montanari 1984)

Polibio. Una polemica contro l'ekphrasis di Filarco è già in Polibio quando dice che essa è più adatta a tragedie che alla storia ${ }^{64}$. L'esempio è la descrizione di Mantinea (223 a.C.) conquistata da Antigono e Arato, cioè una scena dell'urbs capta, tipica per descrizioni.

61 De Martino 2014, 125-134.

62 In 1,5 c'è una sintetica ma efficace descrizione dell'abbigliamento di lusso (calzoni, bracciali e collane) dei nobili militari, pronti a lavori di forza come spingere carri impantanati nella melma. Sui "particolari visivi e d'azione» del racconto di Senofonte, cf. la bella Introduzione di Italo Calvino (1964, 3-9, in part. 3) all'edizione Bur dell'Anabasi.
63 Montanari 1984, 118. Manieri 1998, 162-163 e Berardi 2012, 162 attirano l'attenzione invece su $G l$. Ath. 346f-347c, dove poesia, storiografia e pittura sono messe "tutte sullo stesso piano".

64 Cf. Montanari 1984 (con ulteriore bibliografia), 120, Manieri 1998, 160-162, Berardi 2012, 102, Castellaneta 2013, 113-115. 


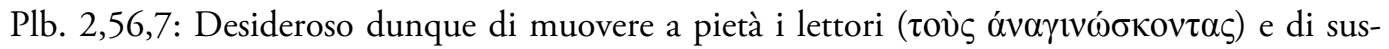
citare la loro simpatia per le sventure narrate, descrive abbracci di donne, capelli strappati, scoper-

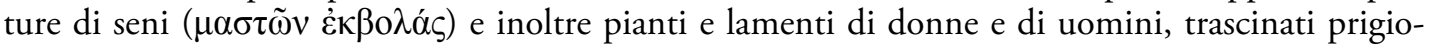
nieri con i figli e i vecchi genitori. Tali rappresentazioni ripete più volte nelle sue Storie sforzandosi di mettere sotto gli occhi ( $\pi \rho$ ò $\tau \tilde{\omega} \nu$ ó$\varphi \theta \alpha \lambda \mu \tilde{\omega} v)$ le cose tremende.

È stato ricordato ${ }^{65}$ tuttavia che, nonostante le sue critiche alle descrizioni, Polibio 5,21,6-7 sostiene l'opportunità di descrivere i luoghi, e dichiara di averlo fatto continuamente nella sua opera. L'ekphrasis dei topoi è caldeggiata soprattutto nelle descrizioni di battaglie, dove si trasforma di conseguenza in ekphrasis mista, perché la battaglia è un pragma. In questo passo programmatico Polibio si inserisce nella discussione dei poeti di teatro su come si descrive una mischia, se cioè ha senso descrivere minutamente i combattenti, nominandoli uno per uno e descrivendone gli scudi. I combattimenti sono scene tipiche della storiografia, come e più che in qualsiasi altro racconto. La priorità dei topoi e della loro conoscenza diretta è esplicitata in 12,25n, dove biasima Timeo perché "assomiglia a quei pittori che copiano da modelli imbottiti di paglia» (Manieri 1998, 158-159; cf. Berardi 2012, 181).

Plb. 5, 21 Perché la mia esposizione, riferendosi a luoghi non molto noti, non riesca vana e confusa occorre che mi soffermi a descriverne la natura e la posizione, come ho cercato di fare in tutto il corso della mia opera, collegando i luoghi sconosciuti a quelli conosciuti o già ricordati. Poiché l'esito dei combattimenti per terra e per mare dipende per lo più dalle posizioni occupate dalle due parti e poiché tutti quanti desideriamo sapere non tanto che cosa sia accaduto, quanto come ogni fatto si sia verificato, non bisogna trascurare in nessun caso di descrivere $i$ luoghi, soprattutto nelle descrizioni delle battaglie, né bisogna esitare a servirsi come punti di riferimento dei porti, dei mari, delle isole, e anche del nome di templi, montagne, regioni e infine della facoltà di orientamento che è comune a tutti gli uomini; solo cosi sarà possibile far comprendere ai lettori quanto essi ignorano. La natura dei luoghi dei quali stiamo trattando è questa:

22 Vista nel suo complesso la pianta di Sparta è circolare e la città è situata in luoghi pianeggianti; considerata nei particolari invece la città comprende zone a terreno irregolare e alture; a oriente scorre il fiume Eurota, per la maggior parte dell'anno inguadabile a causa del volume delle sue acque; le alture sulle quali si leva il Menelaio si trovano al di là del fiume, a sud-est della città, sono aspre, elevate e di difficile accesso, dominano completamente la zona compresa fra il fiume e la città, attraverso la quale, proprio alle falde del colle, scorre l'Eurota; lo spazio intermedio non è più largo di uno stadio e mezzo. (trad. C. Schick)

Non meraviglia che Polibio faccia descrizioni dei topoi, per esempio quella dell' «Italia» e della sua fertilità (2,14 e 15). Meraviglia invece quella dell' "esibizione dei seni» $(15,31,13)$ la stessa che contestava a Filarco.

Agatocle e i suoi, constatando l'ira dei Macedoni e dalle loro azioni e dalla loro risposta, dapprima tentavano di implorare i Macedoni, protendendo attraverso l'inferriata le braccia -e Agatoclea il seno ( re- e ricorrendo a ogni sorta di preghiera per aver salva la vita.

65 Cf. Montanari 1984, 114 che rinvia a Avenarius 1956, 142ss. e Burck 1964², 199ss. con bibliografia. Cf. anche Cic. Or. 66: «Huic generi historia finitima est, in qua narratur ornate et regio saepe aut pugna describitur». 
Agatoclea sorella di Agatocle, il reggente dopo la morte di Tolomeo IV Filopatore (fine III sec. a.C.), implora clemenza esibendo attraverso la grata il seno ${ }^{66}$ che aveva allattato il re bambino, ma del quale poi era divenuta amante. Un nudismo inutile e con destinatario sbagliato perché non rivolto all'allattato. La folla infatti la dilanierà nuda come sta e con la madre e le sorelle.

\section{BiBLIOGRAFIA}

Abbondanza, L. (a cura di), 2008, Filostrato maggiore. Immagini, Milano: Aragno.

Avenarius, G., 1956, Lukians Schrift zur Geschichtsschreibung, Meisenheim-Glan: Hain.

Berardi, F., 2012, La dottrina dell'evidenza nella tradizione retorica greca e latina, Perugia: Pliniana.

Brock van den, R., 1972, The Myth of the Phoenix according to Classical and Early Christian Traditions, Leiden: Brill.

Burck, E., 1964², Die Erzählungskunst des T. Livius, Berlin-Zürich-Weidem.

Calame, C., 1990, "Quand dire c'est faire voir: l'évidence dans la rhétorique antique», Etudes de Lettres, $3-22$.

Calvino, I., 1994, «Introduzione», in: E. Ravenna (ed.), Senofonte. Anabasi, Milano: Rizzoli, 3-9.

Canfora, L., 1974, Teorie e tecniche della storiografia classica, Roma-Bari: Laterza.

Casson, L., 1978, Viaggi e viaggiatori dell'antichità, Mursia, Milano (Travel in the Ancient World, Allen \& Unwin, Ltd, 1974).

Castellaneta, S., 2013, Il seno svelato ad misericordiam. Esegesi e fortuna di un'immagine poetica, Bari: Cacucci.

Cipriani, G., 1986, Cesare e la retorica dell'assedio, J.C. Amsterdam: Gieben.

De Martino, F. 2004, «Il granchio di Chuang-Tzu», Paideia 59, 83-95.

—,2008, «I Greci e l'arte di comunicare», in: S. Nienhaus (ed.), L'attualità della retorica, Bari: Levante, 45-72.

—,2012, «Agamennone re dell'eleganza», in: G. Bastianini, W. Lapini, M. Tulli (eds.), Harmonia. Scritti di filologia classica in onore di Angelo Casanova, I-II, Firenze: Firenze University Press, 2012, 281-293.

-,2013, «Donne di sapere», in: F. De Martino, C. Morenilla, C. (eds.), Palabras sabias de mujeres, Bari: Levante, 111-226.

—,2013, «Ekphrasis e teatro tragico», in: M. Quijada Sagredo, M. C. Encinas Reguero (eds.), Retórica y discurso en el teatro griego, Madrid: Ediciones clásicas, 193-224.

—,2014 «Servi, all'ombra del poeta», in: F. De Martino, C. Morenilla (eds.), A la sombra de los héroes, Bari: Levante, 103-150.

- $2014^{\mathrm{bis}}$, «Tra narrare e descrivere», in A. Ponzio (ed.), Figure e forme del narrare. Incontri di prospettive, Lecce: Milella, 130-143.

Dubel, S., 1997, "Ekphrasis et enargeia: la description antique comme parcours», in: C. Lévy, L. Pernot (eds.), Dire l'évidence (Philosophie et rhétorique antique), Paris-Montreal, 249-264.

Dunbar, N. (ed.), 1995, Aristophanes. Birds, Oxford: Clarendon Press.

Faedo, L., 1994, «Le immagini dal testo. Commento all'apparato iconografico», in: S. Maffei (ed.), Luciano di Samosata. Descrizioni di opere d'arte, Torino: Einaudi.

Fornaro, S., 2002, "Omero cattivo storico. L'orazione XI di Dione Crisostomo», in F. Montanari (a cura di), Omero tremila anni dopo, Roma, Edizioni di Storia e Letteratura, 547-560.

Friedländer, P., 1912, Johannes von Gaza und Paulus Silentiarius, Leipzig-Berlin: Teubner.

Fusillo, M., 1985, Il tempo delle Argonautiche. Un'analisi del racconto in Apollonio Rodio, Roma: Edizioni dell'Ateneo.

${ }^{66}$ Cf. Cipriani 1986, 71, Castellaneta 2013, 114. 
GarzYa, A., 1997, "L'ekphrasis nella tragedia greca», in La parola e la scena. Studi sul teatro antico da Eschilo a Plauto, Napoli, Bibliopolis, 47-58 (= AAP 45, 1996, 87-95).

Grégoire, H., Méridier, L., avec la collaboration de Chapouthier, F., 2002, Euripide. Tragédies, Tome V, Hélène-Les Phéniciennes, Paris: Les Belles Lettres.

Homeyer, H.,1965, Lukian. Wie man Geschichte schreiben soll, München: Fink.

Lloyd, A. L. (ed.), 1989, Erodoto. Le storie, Volume II, Libro II. L'Egitto, Milano: Mondadori.

Longo, O., 1974 1 bis, «Ad Alceo 112, 10 L.P.: per la storia di un topos», Bollettino dell'Istituto di Filologia Greca, Università di Padova 1, 211-228.

—, 1974, "Atene fra polis e territorio: in margine a Tucidide 1,143,5", Studi Italiani di Filologia Classica n.s. 46, pp. 5-21.

—, 1975, «La polis, le mura, le navi (Tucidide, VII, 77, 2)», QS 1, 87-113.

- 1981, Tecniche della comunicazione nella Grecia antica, Napoli: Liguori.

LovisetTo, L., 2005, «La pittura descritta: ह̌ $\kappa \rho \rho \sigma \sigma \varsigma \varsigma$ e riconversioni ecfrastiche nel Quattrocento e Cinquecento», in: M. Centanni (ed.), L'originale assente: introduzione allo studio della tradizione classica, Milano: Bruno Mondadori, 385-402.

Maffei, S. (ed.), 1994, Luciano. Descrizioni di opere d'arte, Torino: Einaudi.

Manieri, A., 1998, L’immagine poetica nella teoria degli antichi. Phantasia ed enargeia, Pisa-Roma: Istituti Editoriali e Poligrafici Internazionali.

Marasco, G., 1978, I viaggi nella Grecia antica, Roma: Ateneo \& Bizzarri.

Mastromarco, G., 1977, «Le mura di Temistocle e le mura di Nubicuculia», QS 6, 1977, 41-50.

Mastromarco, G, Totaro, P., 2006, Commedie di Aristofane, 2, Torino: Utet.

Magistrini, S., 19.70, «Le descrizioni fisiche dei personaggi in Menandro, Plauto e Terenzio», Dioniso 44, 79-114.

Miguélez Cavero, L., 2007, «La Nyktomachia de Trifiodoro: una ekphrasis mixta», in: J.A. Fernández Delgado, F. Pordomingo, A. Stramaglia (eds.), Escuela y Literatura en Grecia Antigua. Actas del Simposio Internacional Universidad de Salamanca 17-19 Noviembre de 2004, Cassino: Edizioni dell'Università degli Studi di Cassino, 497-509.

Montanari, F., 1984, "Ekphrasis e verità storica nella critica di Luciano», in: Ricerche di filologia classica, II, Filologia e critica letteraria della grecità, Pisa: Giardini, 111-123.

Nannini, S., 1986, Omero e il suo pubblico nel pensiero dei commentatori antichi, Roma: Edizioni dell'Ateneo.

Nenci, G. (ed.), 1994, Erodoto. Le Storie, Volume V, La rivolta della Ionia, Milano: Arnoldo Mondadori.

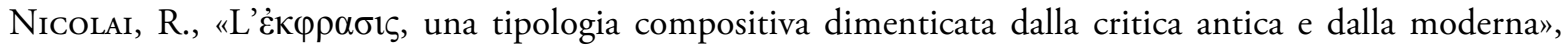
Aion, sez. filol.-lett. 31, 2009, pp. 29-45.

Pavano, G. (ed.), 1958, Dioniso di Alicarnasso. Saggio su Tucidide, Palermo: Priulla.

Quijada Sagredo, M., 2013, "La retórica de la súplica: los discursos de Adrasto y de Etra (Eurípides, Supp. 162-92 y 297-331)», in: M. Quijada Sagredo, M. C. Encinas Reguero (eds.), Retórica y discurso en el teatro griego, Madrid: Ediciones Clásicas, 31-60.

Ravenna, G., 1974, «L'ekphrasis poetica di opere d'arte in latino. Temi e problemi», Quaderni dell'Istituto di Filologia Latina di Padova 3, 1-52.

Ravn, O. E., 1948, Herodotus' Description of Babylon, Kjobenavn: Nyt Nordisk Folrag A. Busck.

Sierra Martín, C., 2014, «Hérodoto (II, 86-88) y el conocimiento anatómico griego", Ágora. Estudios clássicos en debate 16, 29-40.

Zanetto, G. (ed.), 1987, Aristofane. Gli uccelli, Milano: Mondadori.

Zanker, G., 1961, «Enargeia in the Ancient Criticism of Poetry», RhM 124, 297-31. 